\title{
Twin Chorionicity and the Risk of Hypertensive Disorders: Gestational Hypertension and Pre-eclampsia
}

\author{
Pawel Bartnik, ${ }^{1}$ Katarzyna Kosinska-Kaczynska, ${ }^{2}$ Joanna Kacperczyk, ${ }^{1}$ Wojciech Ananicz, ${ }^{1}$ \\ Aleksandra Sierocińska, ${ }^{1}$ Miroslaw Wielgos, ${ }^{2}$ and Iwona Szymusik ${ }^{2}$ \\ ${ }^{1}$ Students' Scientific Association at the 1st Department of Obstetrics and Gynecology, Medical University of Warsaw, \\ Warsaw, Poland \\ 21st Department Of Obstetrics and Gynecology, Medical University of Warsaw, Warsaw, Poland
}

\begin{abstract}
Twin gestation is known to be a risk factor for hypertensive disorders of pregnancy. However, the relationship between hypertensive disorders (pre-eclampsia (PE) and gestational hypertension (GH)) and chorionicity of twin pregnancy is unclear, and published data is conflicting. We decided to analyze the relationship between placentation and prevalence of hypertensive disorders. It was a retrospective cohort study. 312 twin pregnancies delivered between 2009 and 2014 were analyzed, 79 of which were monochorionic and 233 dichorionic. The occurrence of PE and GH was established according to American College of Obstetricians and Gynecologists' (ACOG) guidelines. Hypertensive disorders were diagnosed significantly more often in dichorionic than in monochorionic twin pregnancies $(19.7 \%$ vs. $8.9 \% ; O R=2.53$ $95 \% \mathrm{Cl} 1.04-6.45 ; p=.03)$. PE occurred more frequently in DCP $(13.3 \%$ vs. $3.8 \%$; OR = 3.88 95\% Cl 1.09$16.46 ; p=.02)$. There were no differences between those two groups in the prevalence of $\mathrm{GH}(6.4 \%$ vs. $5.1 \% ; p=.79)$. The logistic regression model for the occurrence of PE included chorionicity, mother's age lower than 18 or higher than 40, pre-gestational obesity, in vitro fertilization, primiparity, gestational age at delivery, gestational diabetes, and active smoking. It showed that dichorionicity remained an independent risk factor for PE (adjusted $O R=4.97 .095 \% \mathrm{Cl} 1.06-23.38 ; p=.04$ ). Dichorionicity seems to be a risk factor for PE but not for GH development.
\end{abstract}

Keywords: twin gestation, preeclampsia, hypertension, chorionicity, multifetal pregnancy, placenta

Twin pregnancies constitute $2-4 \%$ of all births, and their incidence has been rising for the last three decades, especially in the developed countries (Ananth \& Chauhan, 2012). In Poland in 2014, 2.6\% of all births were twin (Central Statistical Office, 2015). Due to an increased use of assisted reproduction techniques (ART) and delayed childbearing, which are associated with a high risk of multiple births (Kupka et al., 2014), the increasing trend in twin pregnancy prevalence is likely to be expected.

Twin gestation is a risk factor for hypertensive disorders of pregnancy. Reported rates of those disorders in twins differ from $13 \%$ to $37 \%$ according to the literature (Long \& Oats, 1987; Ros et al., 1998; Santema et al., 1995). According to Sibai et al. (2000), women with twins have relative risk of 2.04 for developing GH. A systematic review of controlled studies published between 1966 and 2002 revealed that twin pregnancy is associated with 2.93 relative risk of developing PE (Duckitt \& Harrington, 2005). The relationship between $\mathrm{PE}$ and chorionicity is unclear, and published data is conflicting. There are studies indicating that dichorionic pregnancy (DCP) may be at higher (Sarno et al., 2014; Singh et al. 2014, Sparks et al., 2013) or lower risk of PE than monochorionic pregnancy (MCP; Campbell \& MacGillivray, 1999; Campbell \& Templeton, 2004). Other studies (Carter et al., 2015; Leduc et al., 2005; Savvidou et al., 2001) provide information about the lack of similar association.

The aim of the study was to analyze the relationship between chorionicity of twin pregnancy and the risk of both hypertensive disorders of pregnancy: GH and PE.

RECEIVED 26 January 2016; ACCEPTED 24 February 2016. First published online 10 May 2016.

ADDRESS FOR CORRESPONDENCE: Kosinska-Kaczynska Katarzyna, 1st Department Of Obstetrics and Gynecology, Medical University of Warsaw, Starynkiewicza Square 1/3; 02-015 Warsaw, Poland. E-mail: kkaczynska@wum.edu.pl 


\section{Materials and Methods}

A historical cohort study of women in twin pregnancies who delivered at the 1st Department of Obstetrics and Gynecology, Medical University of Warsaw (tertiary university perinatal department) between January 2009 and December 2014 was conducted.

All patients with proper chorionicity assessment who delivered after completed 22 weeks of gestation were included in the study. All the pregnancies were dated according to the last menstrual period or the date of embryo transfer in ART and verified by the crown-rump length measured in the first trimester (in case of crown-rump length discordance, this measurement was taken from the larger twin). Chorionicity was assessed by the ultrasound scan performed in the first trimester of pregnancy on the basis of the number of gestational sacs, embryos, amniotic sacs, membrane thickness, and $\lambda$-sign or $\tau$-sign. If there were no reliable sonography results, twins of a different gender were considered dichorionic.

The presence of PE and GH was assessed according to the ACOG guidelines (ACOG Task Force on Hypertension in Pregnancy, 2013). PE was established by the new onset of hypertension (systolic blood pressure $\geq 140 \mathrm{mmHg}$ or diastolic blood pressure $\geq 90 \mathrm{mmHg}$ on two occasions at least 4 hours apart after 20 weeks of gestation) in a previously normotensive woman, together with proteinuria 0.3 $\mathrm{g} / 24 \mathrm{~h}$ or protein/creatinine ratio $>0.3$. If there was no proteinuria, $\mathrm{PE}$ was diagnosed by the onset of hypertension as above with at least one sign of systemic findings: thrombocytopenia (platelet count $<100.000 /$ microliter), renal insufficiency (serum creatinine $>1.1 \mathrm{mg} / \mathrm{dL}$ or doubling of creatinine in the absence of other renal disease), impaired liver function (liver transaminases at least twice the normal concentrations), pulmonary odema, and new onset of cerebral or visual symptoms. The presence of any of the following features classified PE as severe: systolic blood pressure $\geq 160 \mathrm{mmHg}$ or diastolic blood pressure $\geq 110 \mathrm{mmHg}$ on two occasions at least 4 hours apart while the patient was at bed rest (or under hypertensive therapy), thrombocytopenia (platelet count $<100.000 /$ microliter), impaired liver function (liver transaminases at least twice the normal concentrations and/or severe persistent right upper quadrant or epigastric pain unresponsive to analgesic therapy), progressive renal insufficiency (serum creatinine $>1.1$ $\mathrm{mg} / \mathrm{dL}$ or doubling of creatinine in the absence of other renal disease), pulmonary odema, and new onset of cerebral or visual symptoms. Early onset PE was defined as PE that developed before 34 weeks of gestation, whereas late onset PE developed at or after 34 gestational weeks. The presence of GH was established by the new onset of hypertension (the same diagnostic criteria of hypertension as in PE) without proteinuria or systemic features. Patients with chronic hypertension and glomerulonephritis were excluded from the study due to the difficulties in interpretation of both pos- sible high blood pressure values and proteinuria during pregnancy.

Body mass index (BMI) was calculated by defining the body mass by the square of the body height. Preterm delivery was defined as birth before completed 37 weeks of gestation. Gestational diabetes mellitus (GDM) was diagnosed according to the recommendations of Polish Gynecologic Society (Wender-Ożegowska et al., 2014). Intrauterine fetal demise was defined as fetal death occurring at or after 22 completed gestational weeks. Intrauterine growth restriction (IUGR) was defined as estimated weight below the 10th percentile for the gestational age. Low birth weight (LBW) was defined as birth weight below 2,500 g, very low birth weight (VLBW) as below 1,500 $\mathrm{g}$ and extremely low birth weight (ELBW) as below 1,000 g. Low 1st and 5th minute Apgar scores were defined as 7 points or below.

The cohort was divided into two groups according to chorionicity: MCP twin pregnancy group and DCP twin pregnancy group. Maternal characteristics, GH/PE occurrence and severity, and gestational age at delivery were compared in both analyzed groups. The study outcome was the development of GH or PE in the MCP and DCP groups. The comparative analysis of patients with PE in both groups was also performed. It included maternal characteristics, selected pregnancy complications, and neonatal outcome.

Statistical analysis was performed using Statistica 12 (StatSoft Inc.). Data were presented as means \pm standard deviations or numbers of subjects and percentages. A UMann-Whitney test was used for quantitative data comparison and Fisher's exact test was used for categorical data comparison with a $p$ value $<.05$ considered significant. Eventually, a logistic regression mode for PE adjusted for potential confounding factors was created and odds ratios with $95 \%$ confidence intervals were reported.

\section{Results}

The cohort consisted of 315 women with twin pregnancies. Two patients were excluded due to chronic hypertension and one due to glomerulonephritis, leaving 312 women included in the study. Seventy-nine $(25.3 \%)$ of pregnancies were MCP and 233 (74.7\%) were DCP. The basic characteristics of the analyzed groups are presented in Table 1.

The prevalence of GH and PE in both study groups is presented in Table 2. Hypertensive disorders in pregnancy were diagnosed significantly more often in DCP pregnancies $(19.7 \%$ vs. $8.9 \%$; $p=.03)$, which was mostly due to the higher incidence of $\mathrm{PE}$ in that group (13.3\% vs. $3.8 \%$; $p=$ $.02)$. There were no statistical differences between mild and severe PE, or between early and late onset of PE in the MCP and DCP groups. There were only three cases of PE in the MCP group, all early onset and mild. The incidence of GH did not differ between the analyzed groups. Gestational hypertensive disorders in pregnancy were afterwards stratified by gestational age at delivery into groups $<28$ weeks, 29-31 
TABLE 1

Characteristics of Study Groups

\begin{tabular}{|c|c|c|c|}
\hline Feature & $\mathrm{MCP} N=79$ Mean $\pm S D$ & $\mathrm{DCP} N=233$ Mean $\pm S D$ & $p$ \\
\hline Age (years) & $30.9 \pm 4.9$ & $32.1 \pm 4.2$ & .08 \\
\hline Age $<18^{*}$ & $1(1.3)$ & 0 & .25 \\
\hline Age $>40^{*}$ & $1(1.3)$ & $5(2.1)$ & 1 \\
\hline Pre-pregnancy BMI $\left(\mathrm{kg} / \mathrm{m}^{2}\right)$ & $22.9 \pm 4$ & $22.6 \pm 3.9$ & .49 \\
\hline BMI 25-30* & $18(24)$ & $37(16.7)$ & .17 \\
\hline $\mathrm{BMI} \geq 30^{*}$ & $4(5.3)$ & $9(4.1)$ & .74 \\
\hline Weight gain during pregnancy $(\mathrm{kg})$ & $14.7 \pm 6.7$ & $16.5 \pm 6.2$ & .02 \\
\hline \multicolumn{4}{|l|}{ Gravidity } \\
\hline Primigravidas* & $47(59.5)$ & $139(59.7)$ & \multirow[t]{2}{*}{1} \\
\hline Multigravidas* & $32(40.5)$ & $94(40.3)$ & \\
\hline \multicolumn{4}{|l|}{ Parity } \\
\hline Primaparas* & $50(63.3)$ & $166(71.2)$ & \multirow[t]{2}{*}{.21} \\
\hline Multiparas* & $29(36.7)$ & $67(28.8)$ & \\
\hline Active smokers* & $4(5.6)$ & $16(8.2)$ & .6 \\
\hline $\mathrm{GDM}^{*}$ & $19(25.7)$ & $48(21.8)$ & .52 \\
\hline IVF* & $9(11.4)$ & $109(46.8)$ & $<.001$ \\
\hline Gestational age at delivery (weeks) & $33.8 \pm 3.3$ & $35.2 \pm 3.4$ & $<.001$ \\
\hline Preterm delivery* & $63(79.7)$ & $151(64.8)$ & .02 \\
\hline
\end{tabular}

TABLE 2

Hypertensive Disorders in Pregnancy in Both Study Groups

\begin{tabular}{|c|c|c|c|c|}
\hline Feature & MCP $n=79$ number $(\%)$ & DCP $n=233$ number (\%) & OR $[95 \% \mathrm{Cl}]$ & $p$ \\
\hline \multicolumn{5}{|l|}{$\mathrm{PE} \quad-40$} \\
\hline Any & $3(3.8)$ & $31(13.3)$ & $3.88[1.09-16.46]$ & .02 \\
\hline Mild & $3(3.8)$ & $24(10.3)$ & $2.91[0.8-12.51]$ & .10 \\
\hline Severe & 0 & $7(3)^{*}$ & & .20 \\
\hline Early onset PE & $3(3.8)$ & $23(9.9)$ & $2.78[0.76-11.96]$ & .10 \\
\hline Late onset PE & 0 & $8(3.4)$ & & .21 \\
\hline \multicolumn{5}{|c|}{ PE stratified by gestational age at delivery: } \\
\hline$<28$ weeks & 0 & $1(0.4)$ & & 1 \\
\hline 28-31 weeks & 0 & $2(0.9)$ & & 1 \\
\hline $32-36$ weeks & $3(3.8)$ & $25(10.7)$ & $3.05[0.84-13.05]$ & .07 \\
\hline$\geq 37$ weeks & 0 & $3(1.3)$ & & .57 \\
\hline $\mathrm{GH}$ & $4(5.1)$ & $15(6.4)$ & $1.29[0.39-4.76]$ & .79 \\
\hline \multicolumn{5}{|c|}{ GH stratified by gestational age at delivery: } \\
\hline$<28$ weeks & 0 & 0 & & 1 \\
\hline 28-31 weeks & 0 & 0 & & 1 \\
\hline $32-36$ weeks & $3(3.8)$ & $8(3.4)$ & $0.9[0.21-4.41]$ & 1 \\
\hline$\geq 37$ weeks & $1(1.3)$ & $7(3)$ & $2.42[0.29-53.09]$ & .69 \\
\hline
\end{tabular}

Note: *Including two cases of HELLP syndrome (hemolysis, elevated liver enzymes, low platelet count); MCP = monochorionic twin pregnancy group; DCP = dichorionic twin pregnancy group; $O R=$ odds ratio; $95 \% \mathrm{Cl}=95 \%$ confidence interval; $\mathrm{PE}=$ preeclampsia; $\mathrm{GH}=$ gestational hypertension; weeks = gestational weeks.

weeks, $32-36$ weeks, and $\geq 37$ weeks of gestation. The proportion of DCP with PE increased with gestational age at delivery until 37 weeks, while for MCP, PE occurred only in the 32-36 weeks group. Gestational hypertensive disorders occurred in MCP and DCP pregnancies in the 32-36 weeks and $\geq 37$ weeks group in similar proportions.

Further analysis of pregnancy outcomes of patients with PE in MCP and DCP groups was also performed: $91.2 \%$ of patients with PE delivered preterm (3/3 in MCP and 28/31 in DCP; $p=.8$ ). However, we observed no differences in preterm deliveries ratios between $\mathrm{MCP}$ and $\mathrm{DCP}$, and most of them were late preterm (2/3 in MCP vs. 21/31 in DCP; $p=.8$ ). There was one case of fetal demise in the DCP group due to IUGR and none in the MCP group.
Eventually, the multivariate logistic regression model, which included chorionicity and several confounding factors, was created. Among all analyzed factors only chorionicity and gestational age at delivery were associated with a significant increase or decrease of risk of PE. Results are presented in Table 3. After adjustment, dichorionicity was associated with almost 5-times higher risk of developing PE during pregnancy.

\section{Discussion}

The presented study revealed a significant difference in the prevalence of PE between DCP and MCP. There are only few published studies analyzing this association and their 


\section{TABLE 3}

Multivariate Logistic Regression Model for Pre-eclampsia Adjusted for Potential Confounding Factors

\begin{tabular}{llll}
\hline Parameter & $\begin{array}{l}\text { aOR } \\
\text { (per unit) }\end{array}$ & $\begin{array}{l}95 \% \mathrm{Cl} \\
\text { (per unit) }\end{array}$ & $p$ \\
\hline Chorionicity & 4.97 & $1.06-23.38$ & .04 \\
Gestational age at delivery & 0.86 & $0.74-0.99$ & .03 \\
IVF & 1.8 & $0.95-3.39$ & .07 \\
Age $<18$ or $>40$ years & 1 & $0.11-10.6$ & 1 \\
BMI $\geq 30$ & 1.01 & $0.13-9.12$ & 1 \\
Gestational diabetes mellitus & 2.3 & $0.94-5.61$ & .07 \\
Primiparity & 1.3 & $0.46-3.64$ & .61 \\
Smoking & 1.17 & $0.24-5.72$ & .85 \\
\hline
\end{tabular}

Note: aOR = adjusted odds ratio; $95 \% \mathrm{Cl}=95 \%$ confidence interval; IVF = in vitro fertilization; $\mathrm{BMI}=$ body mass index.

results are conflicting. A few studies indicated no relationship between chorionicity and PE (Carter et al., 2015; Leduc et al., 2005; Savvidou et al., 2001). Two studies reported higher occurrence of PE in MCP than DCP (Campbell \& MacGillivray, 1999; Campbell \& Templeton, 2004). According to Campbell and Templeton (2004), the relative risk for developing PE in MCP was 1.38 [95\% CI 1.04-1.82]. Recent publications indicated a higher incidence of $\mathrm{PE}$ in DCP. Sparks et al. (2013) reported the ratio of PE in the group of 695 twin pregnancies to be significantly higher in DCP than in MCP $(21.1 \%$ vs. $10.8 \%, p<.001)$. A similar association of PE and DCP was confirmed by Sarno et al. (2014) one year later $(30.4 \%$ vs. $12.8 \%, p=.02)$. Singh et al. (2014) also reported a higher incidence of PE in dichorionic and dizygotic twin pregnancies, but their study group was much smaller (208 women) and the results were not significant. In the presented study, the prevalence of PE was lower in both DCP and MCP twins than reported by Sparks et al. (2013) and Sarno et al. (2014). Our results were closer to those published by Singh et al. (2014: 13.2\% vs. 4.9\%). Sparks et al. (2013) also noticed differences in the occurrence of mild PE, which affected significantly more women with DCP, while the incidence of severe PE was similar in both groups. The above was not confirmed in our study, but both differences may be due to a smaller sample size. Nevertheless, it is also important to notice the differences in the revised definition of severe PE, according to the ACOG guidelines (ACOG, 2013). In 2013, massive proteinuria ( $\geq 5 \mathrm{~g}$ ) was eliminated from the diagnostic criteria of severe PE (ACOG; Task Force on Hypertension in Pregnancy, 2013).

A recent study by Carter et al. (2015) showed no differences in PE occurrence in MCP and DCP, which is opposite to our results. Their sample size was bigger (2,301 women); however, there were some differences between our study protocols, which may explain the inconsistency of the results: longer study period (Carter et al. collected data from 1990 to 2010), methods of data collection (Carter et al. lost $10 \%$ of patients in the follow-up between the USG scan and perinatal period) and differences in ethnicity of both groups.
Although we have corrected the risk of PE by considering several potential confounding factors, the association between PE and chorionicity remained significant. Analogous results were reported by Sparks et al. (2013) and Sarno et al. (2014). In our study, the DCP group were at almost 5 times higher risk of developing PE, which was similar to the risk of developing mild PE reported by Sparks et al. (2013; 5.85, 95\% CI 1.31-26.13). Gestational age at delivery was another significant factor influencing the risk of PE. More than half of twin pregnancies were delivered preterm, also due to hypertensive disorders, therefore an increasing gestational age influences the risk of diagnosing PE. The stratification by gestational age performed in the presented analysis revealed that most cases of PE were diagnosed in groups delivering $\geq 32$ weeks of gestation, which was similar to results reported by Sparks et al. (2013). It is important to notice that the majority of cases in our study developed early onset PE. It may be an important observation, as early onset and late onset PE are considered to be separate and pathophysiologically different conditions (Ihle et al., 1987). Whether it is a consequence of a natural tendency for twins to resolve preterm, making it impossible for some of them to develop PE, or whether chorionicity affects the occurrence of early and late onset $\mathrm{PE}$ is not certain and remains an open question. Our study, due to a relatively small sample size, was not able to provide results about this.

We observed no differences in the prevalence of GH between MCP and DCP. To our knowledge, there is only one published study analyzing the occurrence of both $\mathrm{PE}$ and GH in twin pregnancies. Campbell and MacGillivray (1999) reported data on hypertensive disorders in twins with regard to placentation. The incidence of GH was similar in both groups (27.8\% MCP vs. $25.6 \% \mathrm{DCP})$, while the incidence of PE was higher among MCP (20.5\% vs. $14.4 \%)$. Dubé et al. (2002) reported the incidence of pregnancyinduced hypertension in MCP and DCP without analyzing $\mathrm{PE}$ and found no relationship with chorionicity. Our study is the first to analyze both hypertensive disorders in twin pregnancies according to modified ACOG diagnostic criteria. The lack of relationship between GH and chorionicity, confirmed also in our study, sustains suggested differences in etiopathogenesis of $\mathrm{GH}$.

There are two main theories explaining the association between PE and twin placentation. The first one is the increased immunologic response and/or genetic incompatibility theory. Several studies revealed increased concentration of fetal nucleic acids as well as syncytiotrophoblast microparticles in the blood of women affected by PE (Goswami et al., 2006; Hahn et al., 2005). Other studies indicated the presence of maternal natural killer cell interactions with paternal antigens of leukocytes and cytotoxic T-cell response to those antigens as a mechanism leading to the development of PE (de Groot et al., 2010; Stevenson et al., 1971). Women with DCP may be exposed to larger amount of microparticles shed by two placentas and to greater genetic 
variability, which may create more divergent immunological pathways for inappropriate immune response. If immunoincompatibility between the mother and two fetuses was responsible for the development of $\mathrm{PE}$, higher occurrence of $\mathrm{PE}$ should be observed in dizygotic twin pregnancies in comparison to monozygotic ones. However, the increased zygosity has not been found to have a significant effect on PE development in twin pregnancies (Carroll et al., 2005; Maxwell et al., 2001; Savvidou et al., 2001; Singh et al., 2014). In our study group, we were not able to differentiate all monozygotic from dizygotic twins effectively.

The second theory explaining the increased risk of PE in DCP is the placental mass hypothesis. Larger placental mass may be related to an increased release of some molecules with anti-angiogenic activity. Soluble fms-like tyrosine kinase-1 (sFlt-1) was proved to play a crucial role in the pathogenesis of PE (Maynard et al., 2003). The ratio of sFlt-1/PlGF was found to be a good predictor of PE (Bdolah et al., 2008; Dröge et al., 2015; Rana et al., 2012). Several studies showed increased concentrations of sFlt-1 in twin pregnancies affected by PE in comparison to those not affected (Dröge et al., 2015; Levine et al., 2004; Rana et al., 2012) and higher concentrations in twin pregnancies in comparison to singletons (Bdolah et al., 2008). Soluble endoglin (sEng) is another anti-angiogenic factor found to synergize with sFlt-1 in the pathogenesis of maternal syndrome in PE (Venkatesha et al., 2006). Higher concentrations of sEng in twin pregnancies compared to singletons were also reported in the literature (Bdolah et al., 2008; Faupel-Badger et al., 2015). This could suggest the association between the increased placental mass and the risk of preeclampsia. However, no significant differences in placental mass between MCP and DCP were reported in the literature and for this reason the above theory seems to be less possible (Campbell \& MacGillivray, 1999; Gielen et al., 2006). A recent study by Faupel-Badger et al. (Faupel-Badger et al., 2015) also found no correlation between placental mass and sFlt-1, which suggests that greater mass does not explain more anti-angiogenic profile of twin pregnancies. Reduced placental perfusion and hypoxia were proved to increase sFlt-1 production (Nevo et al., 2008). However, as sFlt-1 is a vasoconstrictor and may induce placental ischemia/hypoxia, it is still not clear whether possible placental hypoxia is the effect or the cause of increased antiangiogenic factors release.

Our study is limited by its retrospective nature, a small sample size and selected study group from one tertiary university department. As chorionicity cannot be randomized, the presented study may be prone to confounding bias. We did not investigate a possible correlation between zygosity and hypertensive disorders in pregnancy because zygosity can be determined accurately only by the examination of DNA markers. Finally, as twin pregnancies are often delivered preterm, and monochorionic often earlier than dichorionic ones, it is possible that the rates of $\mathrm{PE}$ and $\mathrm{GH}$ could be different if MCP were continued longer. Nevertheless, after adjusting several confounding factors in multivariate logistic regression model, chorionicity was found to be an independent risk factor of PE.

Dichorionicity seems to be a risk factor for developing preeclampsia, while there is no association between GH and mode of placentation. Further studies on larger population are necessary to determine the relation of different hypertensive disorders in pregnancy and their severity with twin chorionicity.

\section{Acknowledgments}

We would like to gratefully thank Klaudia Wypych for helping in data collection.

\section{Ethical Standards}

The authors assert that all procedures contributing to this work comply with the ethical standards of the relevant national and institutional committees on human experimentation and with the Helsinki Declaration of 1975, as revised in 2008 .

\section{References}

American College of Obstetricians and Gynecologists; Task Force on Hypertension in Pregnancy. (2013). Hypertension in pregnancy: Report of the American college of obstetricians and gynecologists' task force on hypertension in pregnancy. Obstetrics \& Gynecology, 122, 1122-1131.

Ananth, C. V., \& Chauhan, S. P. (2012). Epidemiology of twinning in developed countries. Seminars in Perinatology, 36, $156-161$

Bdolah, Y., Lam, C., Rajakumar, A., Vankatesha, S., Mutter, W., Sachs, B. P., ... Karumanchi, S. A. (2008). Twin pregnancy and the risk of preeclampsia: Bigger placenta or relative ischemia? American Journal of Obstetrics \& Gynecology, 198, 428.e1-428.e6.

Campbell, D. M., \& MacGillivray, I. (1999). Preeclampsia in twin pregnancies: Incidence and outcome. Hypertension in Pregnancy, 18, 197-207.

Campbell, D. M., \& Templeton, A. (2004). Maternal complications of twin pregnancy. International Journal of Gynecology and Obstetrics, 84, 71-73.

Carroll, S. G., Tyfield, L., Reeve, L., Porter, H., Soothill, P., \& Kyle, P. M. (2005). Is zygosity or chorionicity the main determinant of fetal outcome in twin pregnancies? American Journal of Obstetrics \& Gynecology, 193, 757-761.

Carter, E. B., Bishop, K. C., Goetzinger, K. R., Tuuli, M. G., \& Cahill, A. G. (2015). The impact of chorionicity on maternal pregnancy outcomes. American Journal of Obstetrics \& Gynecology, 213, 390.e1-7.

Central Statistical Office. (2015). Demographic yearbook of Poland. Warsaw, Poland: Author.

de Groot, C. J., van der Mast, B. J., Visser, W., De Kuiper, P., Weimar, W., \& Van Besouw, N. M. (2010). Preeclampsia is associated with increased cytotoxic T-cell capacity to 
paternal antigens. American Journal of Obstetrics \& Gynecology, 203, 496.e1-6.

Dröge, L., Herraìz, I., Zeisler, H., Schlembach, D., Stepan, H., Küssel, L.,... Verlohren, S. (2015). Maternal serum sFlt-1/PlGF ratio in twin pregnancies with and without pre-eclampsia in comparison with singleton pregnancies. Ultrasound in Obstetrics \& Gynecology, 45, 286-293.

Dubé, J., Dodds, L., \& Armson, B. A. (2002). Does chorionicity or zygosity predict adverse perinatal outcomes in twins? American Journal of Obstetrics \& Gynecology, 186, 579-583.

Duckitt, K., \& Harrington, D. (2005). Risk factors for preeclampsia at antenatal booking: Systematic review of controlled studies. British Medical Journal, 330, 565.

Faupel-Badger, J. M., McElrath, T. F., Lauria, M., Houghton, L. C., Lim, K. H., Parry, S., ... Troisi, R. (2015). Maternal circulating angiogenic factors in twin and singleton pregnancies. American Journal of Obstetrics \& Gynecology, 212, 636.e1-8.

Gielen, M., Lindsey, P. J., Derom, C., Loos, R. J., Derom, R., Nijhuis, J. G., \& Vlietinck, R. (2006). Curves of placental weights of live-born twins. Twin Research and Human Genetics, 9, 664-672.

Goswami, D., Tannetta, D. S., Magee, L. A., Fuchisawa, A., Redman, C. W., Sargent, I. L., \& von Dadelszen, P. (2006). Excess syncytiotrophoblast microparticle shedding is a feature of early-onset pre-eclampsia, but not normotensive intrauterine growth restriction. Placenta, 27, 56-61.

Hahn, S., Huppertz, B., \& Holzgreve, W. (2005). Fetal cells and cell free fetal nucleic acids in maternal blood: New tools to study abnormal placentation? Placenta, 26, 515-526.

Ihle, B. U., Long, P., \& Oats, J. (1987). Early onset preeclampsia: Recognition of underlying renal disease. British Medical Journal (Clinical Research Ed.), 294, 79-81.

Kupka, M. S., Ferraretti, A. P., de Mouzon, J., Erb, K., D'Hooghe, T., Castilla, J. A., ... Goossens, V. (2014). Assisted reproductive technology in Europe, 2010: Results generated from European registers by ESHRE. Human Reproduction, 29, 2099-2113.

Leduc, L., Takser, L., \& Rinfret, D. (2005). Persistence of adverse obstetric and neonatal outcomes in monochorionic twins after exclusion of disorders unique to monochorionic placentation. American Journal of Obstetrics \& Gynecology, 193, 1670-1675.

Levine, R. J., Maynard, S. E., Qian, C., Lim, K., England, L. J., Yu, K. F., ... Karumanchi, S. A. (2004). Circulating angiogenic factors and the risk of preeclampsia. New England Journal of Medicine, 350, 672-683.

Long, P. A., \& Oats, J. N. (1987). Preeclampsia in twin pregnancy-severity and pathogenesis. Australian and New Zealand Journal of Obstetrics, 27, 1-5.

Maxwell, C. V., Lieberman, E., Norton, M., Cohen, A., Seely, E. W., \& Lee-Parritz, A. (2001). Relationship of twin zygosity and risk of preeclampsia. American Journal of Obstetrics \& Gynecology, 185, 819-821.

Maynard, S. E., Min, J. Y., Merchan, J., Lim, K. H., Li, J., Mondal, S., ... Karumanchi, S. A. (2003). Excess placental soluble fms-like tyrosine kinase 1 (sFlt1) may contribute to endothelial dysfunction, hypertension, and proteinuria in preeclampsia. Journal of Clinical Investigation, 11, 649-658.

Nevo, O., Many, A., Xu, J., Kingdom, J., Piccoli, E., Zamudio, S., ... Caniggia, I. (2008). Placental expression of soluble fmsLike tyrosine kinase 1 is increased in singletons and twin pregnancies with intrauterine growth restriction. Journal of Clinical Endocrinology \& Metabolism, 93, 285-292.

Rana, S., Hacker, M. R., Modest, A. M., Salahuddin, S., Lim, K. H., Verlohren, S., ... Karumanchi, S. A. (2012) Circulating angiogenic factors and risk of adverse maternal and perinatal outcomes in twin pregnancies with suspected preeclampsia. Hypertension, 60, 451-458.

Ros, H. S., Cnattingius, S., \& Lipworth, L. (1998). Comparison of risk factors for preeclampsia and gestational hypertension in a population-based cohort study. American Journal of Epidemiology, 147, 1062-1070.

Santema, J. G., Koppelaar, I., \& Wallenburg, H. C. (1995). Hypertensive disorders in twin pregnancy. European Journal of Obstetrics \& Gynecology and Reproductive Biology, 58, 9-13.

Sarno, L., Maruotti, G. M., Donadono, V., Saccone, G., \& Martinelli, P. (2014). Risk of preeclampsia: Comparison between dichorionic and monochorionic twin pregnancies. Journal of Maternal-Fetal and Neonatal Medicine, 27, 10801081.

Savvidou, M. D., Karanastasi, E., Skentou, C., Geerts, L., \& Nicolaides, K. H. (2001). Twin chorionicity and preeclampsia. Ultrasound in Obstetrics \& Gynecology, 18, 228231.

Sibai, B. M., Hauth, J., Caritis, S., Lindheimer, M. D., MacPherson, C., Klebanoff, M., ... McNellis, D. (2000). Hypertensive disorders in twin versus singleton gestations. National institute of child health and human development network of maternal-fetal medicine units. American Journal of Obstetrics \& Gynecology, 182, 938-942.

Singh, A., Singh, A., Surapaneni, T., \& Nirmalan, P. K. (2014). Pre-eclampsia (PE) and chorionicity in women with twin gestations. Journal of Clinical and Diagnostic Research, 8, 100-102.

Sparks, T. N., Cheng, Y. W., Phan, N., \& Caughey, A. B. (2013). Does risk of preeclampsia differ by twin chorionicity? Journal of Maternal-Fetal and Neonatal Medicine, 26, 12731277.

Stevenson, A. C., Davison, B. C., Say, B., Ustuoplu, S., Liya, D., Abul-Einen, M., \& Toppozada, H. K. (1971). Contribution of fetal/maternal incompatibility to aetiology of preeclamptic toxaemia. Lancet, 2, 1286-1289.

Venkatesha, S., Toporsian, M., Lam, C., Hanai, J., Mammoto, T., Kim, Y. M., ... Karumanchi, S. A. (2006). Soluble endoglin contributes to the pathogenesis of preeclampsia. $\mathrm{Na}$ ture Medicine, 12, 642-649.

Wender-Ożegowska, E., Bomba-Opoń, D., Brazert, J., Celewicz, Z., Czajkowski, K., Karowicz-Blińska, A., ... Zawiejska, A. (2014). Actualization of Polish Gyneacological Society Standards of Medical Care in management of women with diabetes. Ginekologia Polska, 85, 476-478. 